QUARTERLY OF APPLIED MATHEMATICS

VOLUME LXX, NUMBER 2

JUNE 2012, PAGES 253-267

S 0033-569X(2012)01281-0

Article electronically published on February 3, 2012

\title{
GLOBAL EXISTENCE AND ASYMPTOTIC BEHAVIOR FOR A MILDLY DEGENERATE KIRCHHOFF WAVE EQUATION WITH BOUNDARY DAMPING
}

\author{
BY \\ QIONG ZHANG \\ Department of Mathematics, Beijing Institute of Technology, Beijing, 100081, People's Republic of \\ China
}

\begin{abstract}
In this article a degenerate nonlinear dissipative wave equation of Kirchhoff type with nonlinear boundary damping is considered. We prove the existence, uniqueness and regularity of the global solution of the system when the initial data are small enough and the geometry of the domain satisfies suitable assumptions. We also obtain the polynomial decay property of the global solution.
\end{abstract}

1. Introduction. Let $\Omega$ be an open bounded set in $\mathbb{R}^{n}$ with smooth boundary $\Gamma=$ $\Gamma_{0} \cup \Gamma_{1}$. Assume that $\Gamma_{0}$ has positive boundary measure and $\bar{\Gamma}_{0} \cap \bar{\Gamma}_{1}=\emptyset$ (this assumption excludes simply connected regions). We consider the following degenerate nonlinear wave equation of Kirchhoff type with boundary damping:

$$
\left\{\begin{array}{lll}
u^{\prime \prime}(x, t)-M\left(\|\nabla u(x, t)\|^{2}\right) \Delta u(x, t)=0 & \text { in } \quad \Omega \times \mathbb{R}^{+}, \\
u(x, t)=0 & \text { on } \quad \Gamma_{0} \times \mathbb{R}^{+}, \\
M\left(\|\nabla u(x, t)\|^{2}\right) \partial_{\nu} u(x, t)+g\left(u^{\prime}(x, t)\right)=0 & \text { on } \quad \Gamma_{1} \times \mathbb{R}^{+}, \\
u(x, 0)=u_{0}(x), u^{\prime}(x, 0)=u_{1}(x) & \text { in } \quad \Omega,
\end{array}\right.
$$

where $u$ is transverse displacement of the wave, $\|\cdot\|$ represents the norm on $L^{2}(\Omega)$, $\nu$ denotes the unit outward normal vector on boundary, and $M:[0, \infty) \rightarrow[0, \infty)$ is a continuous function. System (1.1) is called a nondegenerate equation when $M(\xi) \geq$ $\tilde{M}>0$ for all $\xi \geq 0$, and a degenerate one when $M(\xi) \geq 0$ for all $\xi \geq 0$. In the case of $M(\xi) \equiv \hat{M}>0$, system (1.1) is a usual linear wave equation with boundary damping.

Received May 4, 2010.

2010 Mathematics Subject Classification. Primary 35L80, 35B40.

Key words and phrases. Degenerate equation, Kirchhoff equation, boundary dissipation, global existence, polynomial decay.

The work is supported by the NSF of China $(60504001,60974033)$, SRF for ROCS, SEM, China (20080732041).

E-mail address: zhangqiong@bit.edu.cn

E-mail address: qiongzhg@gmail.com

(C) 2012 Brown University Reverts to public domain 28 years from publication 
The nonlinear function $g(\cdot)$ is assumed in $C^{1}(R)$, strictly increasing and zero at the origin.

System (1.1) originated in the nonlinear vibration of an elastic string, which was introduced by G. Kirchhoff ([9]) in the 1880s:

$$
u^{\prime \prime}(x, t)-\left(a+b\left\|u_{x}(x, t)\right\|^{2}\right) u_{x x}(x, t)=0 \quad \text { in } \quad(0, L) \times R^{+},
$$

where $a$ and $b$ are positive constants, and $L$ is the length of the string. Later, more general models were considered by Carrier (4]) and Lions ([13]).

Since then, the local and global solvability of nonlinear systems of Kirchhoff type has been studied under various assumptions concerned with the function $M$, dissipative terms and initial data (see, e.g., 1, 6, 8). Among the literature, the latest result about local solvability of a nonlinear system of Kirchhoff type can be found in 8 . They proved the existence, uniqueness and regularity of the local solution for model (1.1) with $k=0$ or $\left.u\right|_{\Gamma}=0$ when $M$ is a degenerate differential function and $M(\xi) \neq 0$ for $\xi$ belonging to a neighborhood of the norm of initial data.

It is clear that an extra dissipative term is necessary to obtain the existence, uniqueness and regularity of the global solution of a nonlinear system of Kirchhoff type. In the nondegenerate case, system (1.1) describes a pre-stressed structure. Brito [3], Nishihara [14] and Cavalcanti [5] proved that there exists a unique global solution with exponential decay property for the system if the initial data is small enough, damping is applied on $\Omega$ and the nondegenerate function $M$ is $C^{1}$-continuous. A similar result was obtained by Lasiecka and Ong [12] if the dissipative term is on the boundary, i.e., the nondegenerate system (1.1).

Degenerate nonlinear systems of Kirchhoff type were considered by Nishihara and Yamada ([15]), Ono ([16]), Ghisi ([7]) and the references therein. They proved that when $M(\xi)$ behaves like $\xi^{\gamma}(\gamma>0)$, a velocity dissipative term is applied in $\Omega$ and the initial data is small and regular enough, there exists a unique global solution of the system and the solution decays with polynomial rate. It is more difficult to handle a degenerate case than a nondegenerate one. Furthermore, the difficulty increases when the damping is applied on the boundary. The method in the references cannot be applied directly to the degenerate Kirchhoff system with boundary damping.

In this paper we study the global existence and decay properties of the solution of a degenerate Kirchhoff wave equation system with nonlinear boundary damping. The nonlinear coefficient function is assumed to be $M(\xi)=\xi^{\gamma}(\gamma>1)$. To prove the existence, uniqueness and regularity of the global solution of the system, we first introduce estimates of higher order energies divided by $\|\nabla u\|^{s}(s>0)$ to overcome difficulties due to the degenerateness of $M(\xi)$. Then by the idea of iterativeness of the time and assumptions on the initial data, we show that $\|\nabla u(t)\|>0$ for all $t>0$, i.e., the degenerateness situation never does occur because of the dissipation. Thus, the local solution of system (1.1) can be continued globally in time. We also obtain the polynomial decay of the solution of system (1.1) by the classical Gronwall lemma.

Our paper is organized as follows. In Section 2 we present the local existence of the solution of the system. In Section 3 we prove the existence, uniqueness and regularity of the global solution. The decay property of the solution is given in Section 4 . 
2. Preliminaries. In what follows, $H^{r}(\Omega)$ denotes the usual Sobolev space for any $r \in \mathbb{R}$. $H_{\Gamma_{0}}^{1}(\Omega)$ denotes the space $\left\{w \in H^{1}(\Omega): w=0\right.$ on $\left.\Gamma_{0}\right\}$. Let $X$ be a Banach space. We denote by $C^{m}([0, T] ; X)$ the space of all $m$ times continuously differentiable functions defined on $[0, T]$ with values in $X$, and write $C([0, T] ; X)$ for $C^{0}([0, T] ; X)$. The scalar product and norm in $L^{2}(\Omega)$ and $L^{2}(\Gamma)$ are represented by $\|\cdot\|,(\cdot, \cdot)$ and $|\cdot|_{\Gamma}$, $\langle\cdot, \cdot\rangle_{\Gamma}$, respectively.

In this paper we consider the global existence and decay property of the solution of the following degenerate Kirchhoff wave equation with boundary damping:

$$
\left\{\begin{array}{lll}
u^{\prime \prime}(x, t)-\|\nabla u(x, t)\|^{2 \gamma} \Delta u(x, t)=0 & \text { in } & \Omega \times \mathbb{R}^{+}, \\
u(x, t)=0 & \text { on } & \Gamma_{0} \times \mathbb{R}^{+}, \\
\|\nabla u(x, t)\|^{2 \gamma} \partial_{\nu} u(x, t)+g\left(u^{\prime}(x, t)\right)=0 & \text { on } & \Gamma_{1} \times \mathbb{R}^{+}, \\
u(x, 0)=u_{0}(x), u^{\prime}(x, 0)=u_{1}(x) & \text { in } & \Omega .
\end{array}\right.
$$

We first present assumptions on the function $g(\cdot)$ and the geometry of the domain.

(G) $g(0)=0, c_{1} \leq g^{\prime}(s) \leq c_{2}, c_{3}|s| \leq|g(s)| \leq c_{4}|s|, c_{i}>0, i=1,2,3,4$.

(B1) $m(x) \cdot \nu(x) \leq 0$ on $\Gamma_{0}$, where $m(x) \doteq x-x_{0}$, and $x_{0}$ is an arbitrary fixed point in $\mathbb{R}^{n}$.

The natural energy of system (2.1) is defined by

$$
E(t)=\frac{1}{2}\left\|u^{\prime}(t)\right\|^{2}+\frac{1}{2 \gamma+2}\|\nabla u(t)\|^{2 \gamma+2} .
$$

It is clear from $(\mathrm{G})$ that

$$
\frac{d}{d t} E(t)=-\left\langle g\left(u^{\prime}(t)\right), u^{\prime}(t)\right\rangle_{\Gamma_{1}} \leq 0,
$$

i.e., $E(t)$ decreases on $[0, \infty)$.

By applying the Banach contraction mapping theorem, we get the following local existence theorem.

Theorem 2.1. Let $\gamma>0, g(\cdot)$ satisfy $(\mathrm{G})$ and the initial data $u_{0} \in H^{2}(\Omega) \cap H_{\Gamma_{0}}^{1}(\Omega)$, $u_{1} \in H_{\Gamma_{0}}^{1}(\Omega)$. Suppose $\left\|\nabla u_{0}\right\|>0$. Then there exists $T=T\left(\left\|\nabla u_{0}\right\|\right)>0$ such that system (2.1) has a unique local solution $u(x, t)$ with regularity

$$
u \in C\left([0, T] ; H^{2}(\Omega) \cap H_{\Gamma_{0}}^{1}(\Omega)\right) \cap C^{1}\left([0, T] ; H_{\Gamma_{0}}^{1}(\Omega)\right) \cap C^{2}\left([0, T] ; L^{2}(\Omega)\right) .
$$

Proof. First, we consider the functional space

$$
\begin{gathered}
X_{T, L} \doteq\left\{w(t) \in C\left([0, T] ; H^{2}(\Omega) \cap H_{\Gamma_{0}}^{1}(\Omega)\right) \cap C^{1}\left([0, T] ; H_{\Gamma_{0}}^{1}(\Omega)\right): w(0)=u_{0},\right. \\
\left.w^{\prime}(0)=u_{1},\|\nabla w(t)\|^{2},\left\|\nabla w^{\prime}(t)\right\|^{2},\|\Delta w(t)\|^{2} \leq L \text { on }[0, T]\right\},
\end{gathered}
$$

where $T>0$ and $L>0$ satisfy

$$
T=\frac{M_{0}}{4 L}, \quad M_{0} \doteq\left\|\nabla u_{0}\right\|^{2} .
$$

Then, for any $w \in X_{T, L}$, we have by the Lagrange theorem that

$$
\|\nabla w(t)\|^{2} \geq \frac{M_{0}}{2}, \quad \forall t \in[0, T] .
$$


Define a map $\mathcal{S}$ as follows. For $w \in X_{T, L}, u=\mathcal{S} w$ is the unique solution of the following linear problem:

$$
\left\{\begin{array}{lll}
u^{\prime \prime}(x, t)-\|\nabla w(x, t)\|^{2 \gamma} \Delta u(x, t)=0 & \text { in } & \Omega \times \mathbb{R}^{+}, \\
u(x, t)=0 & \text { on } & \Gamma_{0} \times \mathbb{R}^{+}, \\
\|\nabla w(x, t)\|^{2 \gamma} \partial_{\nu} u(x, t)+g\left(u^{\prime}(x, t)\right)=0 & \text { on } & \Gamma_{1} \times \mathbb{R}^{+}, \\
u(x, 0)=u_{0}(x), u^{\prime}(x, 0)=u_{1}(x) & \text { in } & \Omega .
\end{array}\right.
$$

Now we prove that $\mathcal{S}$ maps $X_{T, L}$ into itself if (2.5) holds. By multiplying the first equation of system (2.7) by $2 u^{\prime}$ and integrating it over $\Omega$, we have

$$
\begin{aligned}
\frac{d}{d t}\left(\left\|u^{\prime}(t)\right\|^{2}+\|\nabla w(t)\|^{2 \gamma}\|\nabla u(t)\|^{2}\right)= & 2 \gamma\left(\nabla w(t), \nabla w^{\prime}(t)\right)\|\nabla w(t)\|^{2 \gamma-2}\|\nabla u(t)\|^{2} \\
& -2\left\langle g\left(u^{\prime}(t)\right), u^{\prime}(t)\right\rangle_{\Gamma_{1}} .
\end{aligned}
$$

It follows from $(\mathrm{G}),(2.6)$ and (2.8) that

$$
\frac{d}{d t}\left(\left\|u^{\prime}(t)\right\|^{2}+\|\nabla w(t)\|^{2 \gamma}\|\nabla u(t)\|^{2}\right) \leq 4 \gamma L M_{0}^{-1}\|\nabla w(t)\|^{2 \gamma}\|\nabla u(t)\|^{2}, \quad \forall 0 \leq t \leq T .
$$

Thus, we have from the Gronwall inequality that for all $0<t \leq T$,

$$
\left\|u^{\prime}(t)\right\|^{2}+\|\nabla w(t)\|^{2 \gamma}\|\nabla u(t)\|^{2} \leq\left(\left\|u_{1}\right\|^{2}+\left\|\nabla u_{0}\right\|^{2 \gamma+2}\right) e^{\varepsilon_{1} L T}, \quad \varepsilon_{1} \doteq 4 \gamma M_{0}^{-1} .
$$

Consequently,

$$
\left\|u^{\prime}(t)\right\|^{2},\|\nabla u(t)\|^{2} \leq \max \left\{1,2^{\gamma} M_{0}^{-\gamma}\right\}\left(\left\|u_{1}\right\|^{2}+\left\|\nabla u_{0}\right\|^{2 \gamma+2}\right) e^{\varepsilon_{1} L T}, \quad \forall 0 \leq t \leq T .
$$

Moreover, we multiply the first equation of (2.7) by $2 \Delta u^{\prime}$ and integrate it over $\Omega$,

$$
\begin{aligned}
\frac{d}{d t} F(t)= & 2 \gamma\left(\nabla w(t), \nabla w^{\prime}(t)\right)\left[\|\nabla w\|^{2 \gamma-2}\|\Delta u(t)\|^{2}+\frac{2\left\langle u^{\prime \prime}(t), g\left(u^{\prime}(t)\right)\right\rangle_{\Gamma_{1}}}{\|\nabla w(t)\|^{2 \gamma+2}}\right] \\
& -\frac{2\left\langle g^{\prime}\left(u^{\prime}(t)\right) u^{\prime \prime}(t), u^{\prime \prime}(t)\right\rangle_{\Gamma_{1}}}{\|\nabla w(t)\|^{2 \gamma}}
\end{aligned}
$$

where $F(t) \doteq\left\|\nabla u^{\prime}(t)\right\|^{2}+\|\nabla w(t)\|^{2 \gamma}\|\Delta u(t)\|^{2}$. Thus, by using (G) and the CauchySchwarz inequality,

$$
\frac{d}{d t} F(t) \leq 2 \gamma\left(\nabla w(t), \nabla w^{\prime}(t)\right)\|\nabla w(t)\|^{2 \gamma-2}\|\Delta u(t)\|^{2}+\frac{2 \gamma^{2}\left(\nabla w(t), \nabla w^{\prime}(t)\right)^{2}}{c_{1}\|\nabla w(t)\|^{2 \gamma+4}}\left|g\left(u^{\prime}(t)\right)\right|_{\Gamma_{1}}^{2} .
$$

Note that by the trace theorem, there exists a positive constant $C_{\Omega}$ such that

$$
\left|u^{\prime}\right|_{\Gamma_{1}}^{2} \leq C_{\Omega}\left\|\nabla u^{\prime}\right\|^{2} .
$$

Combining (G), (2.13) with (2.14) yields

$$
\frac{d}{d t} F(t) \leq 2 \gamma\left\|\nabla w^{\prime}(t)\right\|\|\nabla w(t)\|^{2 \gamma-1}\|\Delta u(t)\|^{2}+\frac{2 c_{4}^{2} C_{\Omega} \gamma^{2}\left\|\nabla w^{\prime}(t)\right\|^{2}\left\|\nabla u^{\prime}(t)\right\|^{2}}{c_{1}\|\nabla w(t)\|^{2 \gamma+2}} .
$$

Thus, by (2.6) and (2.15),

$$
\frac{d}{d t} F(t) \leq \varepsilon_{2} L F(t), \quad \forall 0 \leq t \leq T,
$$


where

$$
\varepsilon_{2} \doteq \max \left\{\varepsilon_{1}, 2^{\gamma+2} c_{4}^{2} C_{\Omega} \gamma^{2} c_{1}^{-1} M_{0}^{-(\gamma+1)}\right\}
$$

Therefore,

$$
\left\|\nabla u^{\prime}(t)\right\|^{2}, \quad\|\Delta u(t)\|^{2} \leq \max \left\{1,2^{\gamma} M_{0}^{-\gamma}\right\} F(0) e^{\varepsilon_{2} L T}, \quad \forall 0 \leq t \leq T .
$$

Set

$$
L \doteq M^{*} \max \left\{1,2^{\gamma} M_{0}^{-\gamma}\right\}\left(\left\|u_{1}\right\|^{2}+\left\|\nabla u_{0}\right\|^{2 \gamma+2}+\left\|\nabla u_{1}\right\|^{2}+\left\|\nabla u_{0}\right\|^{2 \gamma}\left\|\Delta u_{0}\right\|^{2}\right),
$$

where

$$
M^{*} \geq e^{\frac{\varepsilon_{2} M_{0}}{4}} .
$$

Hence, we obtain that $\mathcal{S}$ maps $X_{T, L}$ into itself from (2.11) and (2.17) if $T$ and $L$ satisfy (2.5) and (2.18).

By a similar technique, we can prove the contraction property of map $\mathcal{S}$. Thus, by the contraction mapping principle, we obtain that there exists a unique local solution $u(x, t)$ of system (2.1) with smooth initial data $u_{0} \in H^{2}(\Omega) \cap H_{\Gamma_{0}}^{1}(\Omega), u_{1} \in H_{\Gamma_{0}}^{1}(\Omega)$, and $u(x, t)$ satisfies (2.4).

3. Global existence. In this section we shall consider the global existence of the solution of system (2.1). The main result of this section is as follows.

Theorem 3.1. Assume (G) and (B1) hold, $\gamma \geq 1$. Let the initial data $u_{0} \in H^{2}(\Omega) \cap$ $H_{\Gamma_{0}}^{1}(\Omega), u_{1} \in H_{\Gamma_{0}}^{1}(\Omega)$ satisfy

$$
\left\|\nabla u_{0}\right\|>0
$$

and

$$
\left\|\nabla u_{0}\right\|^{2 \gamma}\left\|\Delta u_{0}\right\|^{2}+\left\|\nabla u_{1}\right\|^{2}+\frac{\left\|\Delta u_{0}\right\|^{2}}{\left\|\nabla u_{0}\right\|^{2 \gamma+2}}+\frac{\left\|\nabla u_{1}\right\|^{2}}{\left\|\nabla u_{0}\right\|^{4 \gamma+2}}<\kappa,
$$

where $\kappa$ is a given positive constant. Then system (2.1) has a unique global solution $u(x, t)$ with regularity

$$
u \in C\left([0, \infty) ; H^{2}(\Omega) \cap H_{\Gamma_{0}}^{1}(\Omega)\right) \cap C^{1}\left([0, \infty) ; H_{\Gamma_{0}}^{1}(\Omega)\right) \cap C^{2}\left([0, \infty) ; L^{2}(\Omega)\right) .
$$

REMARK 3.1. In Theorem 3.1 $\kappa$ is a constant depending on $\Omega, g(\cdot)$ and $\gamma$. More precisely, $\kappa$ satisfies

$$
\kappa<\frac{\beta^{2}}{4 \lambda}
$$

where $\beta>0$ satisfies (3.14) and $\lambda$ is defined by (3.18).

To prove Theorem 3.1, we need to introduce several auxiliary functions. Since $\left\|\nabla u_{0}\right\|>$ 0 , we can define

$$
T_{1} \doteq \sup \{t \in[0, \infty):\|\nabla u(s)\|>\alpha, \quad \forall 0 \leq s<t\},
$$


where $\alpha$ is a sufficiently small positive constant. Then, for $0 \leq t \leq T_{1}$, we set

$$
\begin{aligned}
& H_{1}(t) \doteq\|\nabla u(x, t)\|^{2 \gamma}\|\Delta u(x, t)\|^{2}+\left\|\nabla u^{\prime}(x, t)\right\|^{2}, \\
& H_{2}(t) \doteq \frac{\|\Delta u(x, t)\|^{2}}{\|\nabla u(x, t)\|^{2 \gamma+2}}+\frac{\left\|\nabla u^{\prime}(x, t)\right\|^{2}}{\|\nabla u(x, t)\|^{4 \gamma+2}}, \\
& \rho(t) \doteq\left(\Delta u(x, t), 2 m(x) \cdot \nabla u^{\prime}(x, t)\right)+(n-1)\left(\Delta u(x, t), u^{\prime}(x, t)\right), \\
& \Phi_{p}(t) \doteq \frac{\left\|\nabla u^{\prime}(x, t)\right\|}{\|\nabla u(x, t)\|^{p}}, \quad p \in \mathbb{N} .
\end{aligned}
$$

It is clear from (2.3) that for any $0 \leq t<T_{1}$,

$$
|\rho(t)| \leq \rho_{0} H_{2}(t)
$$

where

$$
\begin{aligned}
& \rho_{0} \doteq e_{0}^{2 \gamma+2}+\left[R^{2}+\frac{(n-1)^{2}}{4}\right] e_{0}^{4 \gamma+2}, \\
& R \doteq \max _{x \in \bar{\Omega}}|m(x)|, \\
& e_{0} \doteq[(2 \gamma+2) E(0)]^{\frac{1}{2 \gamma+2}} .
\end{aligned}
$$

We can get the following properties of $H_{1}$ and $H_{2}$ by direct computation.

Lemma 3.1. Let $u(x, t)$ satisfy system (2.1) and let assumption (G) hold. Then, for any $0 \leq t<T_{1}$

$$
\begin{gathered}
\frac{d}{d t} H_{1}(t) \leq-\frac{c_{1}\left|u^{\prime \prime}(t)\right|_{\Gamma_{1}}^{2}}{\|\nabla u(t)\|^{2 \gamma}}+\frac{4 \gamma^{2}}{c_{1}} \Phi_{\gamma+1}^{2}(t)\left|g\left(u^{\prime}(t)\right)\right|_{\Gamma_{1}}^{2}+2 \gamma \Phi_{1}(t) H_{1}(t), \\
\frac{d}{d t} H_{2}(t) \leq-\frac{c_{1}\left|u^{\prime \prime}(t)\right|_{\Gamma_{1}}^{2}}{\|\nabla u(t)\| \|^{6 \gamma+2}}+\frac{4 \gamma^{2}}{c_{1}} \Phi_{3 \gamma+2}^{2}(t)\left|g\left(u^{\prime}(t)\right)\right|_{\Gamma_{1}}^{2}+(4 \gamma+2) \Phi_{1}(t) H_{2}(t) .
\end{gathered}
$$

The following lemma is useful and its proof is given in the Appendix.

Lemma 3.2. Assume (G) and (B1) hold, $\gamma \geq 1$. Let $u(x, t)$ satisfy system (2.1). Then, for any $0 \leq t<T_{1}$,

$$
\begin{aligned}
& \int_{0}^{t} H_{1}(s) d s \\
& \leq \quad C_{0} H_{1}(0)+2 \rho_{0} H_{2}(0)+\int_{0}^{t}\left\{\frac{R\left|u^{\prime \prime}(s)\right|_{\Gamma_{1}}^{2}}{\|\nabla u(s)\|^{2 \gamma}}+\frac{2 C_{B}\left|g^{\prime}\left(u^{\prime}(s)\right) u^{\prime \prime}(s)\right|_{\Gamma_{1}}^{2}}{\|\nabla u(s)\|^{4 \gamma}}+2 R C_{\delta, \epsilon}\left|u^{\prime \prime}(s)\right|_{\Gamma_{1}}^{2}\right. \\
& \quad+\frac{\left|u^{\prime}(s)\right|_{\Gamma_{1}}^{2}}{4 C_{\Omega}}+\frac{8 \gamma^{2}}{c_{1}}\left[c_{1} C_{B} \Phi_{2 \gamma+1}^{2}(s)+\delta \Phi_{\gamma+1}^{2}(s)+\rho_{0} \Phi_{3 \gamma+2}^{2}(s)\right]\left|g\left(u^{\prime}(s)\right)\right|_{\Gamma_{1}}^{2} \\
& \left.\quad+\left[4 \gamma \delta \Phi_{1}(s)+2(4 \gamma+2) \rho_{0} \Phi_{4 \gamma+3}(s)+8 \gamma^{2} R C_{\delta, \epsilon} e_{0}^{2 \gamma} \Phi_{1}^{2}(s)\right] H_{1}(s)\right\} d s
\end{aligned}
$$

where $0<\delta<\frac{t}{2}, \epsilon, \epsilon_{0}>0$ are arbitrary, $C_{\delta, \epsilon}, C_{\delta, \epsilon_{0}}, C_{t}$ are positive constants, and

$$
\begin{aligned}
& C_{0}=2 \delta+2 R C_{\delta, \epsilon_{0}} C_{t}\left(1+e_{0}^{2 \gamma}\right), \\
& C_{B}=3 R+2 R C_{\delta, \epsilon}+(n-1)^{2} C_{\Omega}+2 R C_{\delta, \epsilon_{0}} C_{t} .
\end{aligned}
$$


Now we prove Theorem 3.1, Set

$$
\mathcal{E}(t) \doteq \lambda_{1} H_{1}(t)+\lambda_{2} H_{2}(t)
$$

where $\lambda_{i}(i=1,2,3)$ are positive constants satisfying

$$
\begin{aligned}
& \lambda_{1} \geq \max \left\{\frac{R\left(1+2 C_{\delta, \epsilon} e_{0}^{2 \gamma}\right)}{c_{1}}, C_{0}\right\}, \\
& \lambda_{2} \geq \max \left\{\frac{2 c_{2}^{2} C_{B} e_{0}^{2 \gamma+2}}{c_{1}}, 2 \rho_{0}\right\} .
\end{aligned}
$$

Then, we have from $(\mathrm{G})$, Lemmas $3.1+3.2$ that

$$
\begin{aligned}
\mathcal{E}(t) & +\int_{0}^{t} H_{1} d s \\
& \leq 2 \mathcal{E}(0)+\int_{0}^{t}\left\{\frac{\left|u^{\prime}\right|_{\Gamma_{1}}^{2}}{4 C_{\Omega}}+\frac{4 \gamma^{2} c_{4}^{2}}{c_{1}}\left[2 c_{1} C_{B} \Phi_{2 \gamma+1}^{2}+\left(2 \delta+\lambda_{1}\right) \Phi_{\gamma+1}^{2}+\left(2 \rho_{0}+\lambda_{2}\right) \Phi_{3 \gamma+2}^{2}\right]\left|u^{\prime}\right|_{\Gamma_{1}}^{2}\right. \\
& \left.+\left[2 \gamma\left(2 \delta+\lambda_{1}\right) \Phi_{1}+(4 \gamma+2)\left(2 \rho_{0}+\lambda_{2}\right) \Phi_{4 \gamma+3}+8 \gamma^{2} R C_{\delta, \epsilon} e_{0}^{2 \gamma} \Phi_{1}^{2}\right] H_{1}\right\} d s .9
\end{aligned}
$$

Moreover, for a positive constant $\beta$, define

$$
T_{2} \doteq \sup \left\{t \in[0, \infty): \Phi_{2 \gamma+1}(s)<\beta, \quad \forall 0 \leq s<t\right\} .
$$

It is clear that $T_{2}>0$. Suppose

$$
T_{2}<T_{1} .
$$

Then,

$$
\Phi_{2 \gamma+1}\left(T_{2}\right)=\beta .
$$

It follows from (2.3), (2.14) and (3.10) that for any $0 \leq t \leq T_{2}$,

$$
\mathcal{E}(t)+\int_{0}^{t} H_{1}(s) d s \leq 2 \mathcal{E}(0)+\int_{0}^{t}\left(\frac{1}{4}+\mu_{1} \beta+\mu_{2} \beta^{2}\right) H_{1}(s) d s,
$$

where

$$
\begin{aligned}
& \mu_{1} \doteq 2 \gamma\left(2 \delta+\lambda_{1}\right) e_{0}^{2 \gamma}+\frac{(4 \gamma+2)\left(2 \rho_{0}+\lambda_{2}\right)}{\alpha^{2 \gamma+2}} \\
& \mu_{2} \doteq \frac{4 \gamma^{2} c_{4}^{2} C_{\Omega}}{c_{1}}\left[2 c_{1} C_{B}+\left(2 \delta+\lambda_{1}\right) e_{0}^{2 \gamma}+\frac{2 \rho_{0}+\lambda_{2}}{\alpha^{2 \gamma+2}}\right]+8 \gamma^{2} R C_{\delta, \epsilon} e_{0}^{6 \gamma}
\end{aligned}
$$

Let $\beta$ satisfy

$$
0<\beta<\frac{-\mu_{1}+\left(\mu_{1}^{2}+\mu_{2}\right)^{\frac{1}{2}}}{2 \mu_{2}} .
$$

Then,

$$
\mu_{1} \beta+\mu_{2} \beta^{2}<\frac{1}{4}
$$

Thus, it follows from (3.13)-(3.15) that

$$
\mathcal{E}(t) \leq 2 \mathcal{E}(0), \quad \forall 0 \leq t \leq T_{2} .
$$

Consequently, for any $0 \leq t \leq T_{2}$,

$$
H_{1}(t)+H_{2}(t) \leq \lambda\left[H_{1}(0)+H_{2}(0)\right]
$$


where

$$
\lambda \doteq \frac{2 \max \left\{\lambda_{1}, \lambda_{2}\right\}}{\min \left\{\lambda_{1}, \lambda_{2}\right\}}
$$

On the other hand, it is clear that for any $0 \leq t<T_{1}$,

$$
\Phi_{2 \gamma+1}(t) \leq H_{2}^{\frac{1}{2}}(t)
$$

Combining (3.11), (3.17) with (3.19) yields that for any $0 \leq t \leq T_{2}<T_{1}$,

$$
\Phi_{2 \gamma+1}(t) \leq\left[\lambda\left(H_{1}(0)+H_{2}(0)\right)\right]^{\frac{1}{2}} .
$$

Therefore, from Remark 3.1, we have from (3.20) that

$$
\Phi_{2 \gamma+1}(t)<\frac{\beta}{2}, \quad 0 \leq t \leq T_{2} .
$$

We get the contradiction to (3.12). Therefore, $T_{1} \leq T_{2}$.

$t \geq 0$. By the definition of $T_{1}$, we have that

$$
\left\|\nabla u\left(T_{1}\right)\right\|=0 .
$$

Since $T_{1} \leq T_{2}$, we have that estimation (3.17) holds when $0 \leq t \leq T_{1}$. Then we have from (3.22) that

$$
\left\|\nabla u^{\prime}\left(T_{1}\right)\right\|=0 .
$$

Induce a variable $v(t) \doteq u\left(T_{1}-t\right)$. Then $v(t)$ satisfies

$$
\left\{\begin{array}{lll}
v^{\prime \prime}-\|\nabla v\|^{2 \gamma} \Delta v=0 & \text { in } & \Omega \times\left[0, T_{1}\right], \\
v=0 & \text { on } & \Gamma_{0} \times\left[0, T_{1}\right], \\
\|\nabla v\|^{2 \gamma} \partial_{\nu} v+g\left(-v^{\prime}\right)=0 & \text { on } & \Gamma_{1} \times\left[0, T_{1}\right], \\
v(0)=0, v^{\prime}(0)=0 & \text { in } & \Omega .
\end{array}\right.
$$

It is clear that

$$
\frac{d}{d t} E(v(t))=-\left\langle g\left(-v^{\prime}(t)\right), v^{\prime}(t)\right\rangle_{\Gamma_{1}}
$$

Moreover, from (3.17),

$$
\left\|\nabla u^{\prime}\left(T_{1}-t\right)\right\|^{2} \leq \lambda \mathcal{E}(0)\left\|\nabla u\left(T_{1}-t\right)\right\|^{4 \gamma+2} .
$$

Hence, by the trace theorem and (3.25)-(3.26), we get that

$$
\frac{d}{d t} E(v(t)) \leq c_{4} C_{\Omega} \lambda \mathcal{E}(0) e_{0}^{2 \gamma}(2 \gamma+2) E(v(t)), \quad \forall 0 \leq t \leq T_{1} .
$$

Notice that $E(v(0))=0$. Thus, it follows from (3.27) that $E\left(v\left(T_{1}\right)\right)=0$. Consequently, $\|\nabla u(0)\|=0$, which contradicts assumption (3.1). Therefore, $\|\nabla u(t)\|>0$ for all $t \geq 0$, and (3.17) holds for all $t \geq 0$. The proof is completed. 
4. Polynomial decay. In this section, we shall study the asymptotic behavior of the global solution of system (2.1). First, we introduce the following geometry assumption on domain $\Omega$.

(B2) $m(x) \cdot \nu(x) \geq m_{0}>0$ on $\Gamma_{1}$.

The main result of this section is as follows.

Theorem 4.1. Assume (G), (B1) and (B2) hold, $\gamma \geq 1$. Let the initial data $u_{0} \in H^{2}(\Omega) \cap$ $H_{\Gamma_{0}}^{1}(\Omega), u_{1} \in H_{\Gamma_{0}}^{1}(\Omega)$ satisfy $\left\|\nabla u_{0}\right\|>0$ and

$$
\left\|\nabla u_{0}\right\|^{2 \gamma}\left\|\Delta u_{0}\right\|^{2}+\left\|\nabla u_{1}\right\|^{2}+\frac{\left\|\Delta u_{0}\right\|^{2}}{\left\|\nabla u_{0}\right\|^{2 \gamma+2}}+\frac{\left\|\nabla u_{1}\right\|^{2}}{\left\|\nabla u_{0}\right\|^{4 \gamma+2}}<\tilde{\kappa},
$$

where $\tilde{\kappa}$ is a given positive constant. Then there exists a positive constant $C_{E}$ depending on $\Omega, g(\cdot), \gamma$ and the initial data such that

$$
E(t) \leq \frac{C_{E}}{(1+t)^{\frac{2 \gamma+2}{\gamma}}}, \quad \forall t \geq 0 .
$$

REMARK 4.1. In (4.1), the constant $\tilde{\kappa}$ satisfies

$$
\tilde{\kappa} \leq \min \left\{\kappa, \frac{1}{4 \eta \lambda C_{\Omega}(\gamma+1)}\right\},
$$

where $\kappa, \eta, \lambda$ and $C_{\Omega}$ are defined by Remark 3.1, (4.7), (3.18) and (2.14), respectively.

In (4.2), the constant $C_{E}$ is defined as

$$
C_{E} \doteq\left(\tilde{\lambda}-\tilde{\rho}_{0}\right)^{\frac{(1-\theta)(2 \gamma+2)}{\gamma}} C_{\mathcal{E}}^{\frac{2 \gamma+2}{\gamma}},
$$

where $\tilde{\rho}_{0}, \tilde{\lambda}, \theta$ and $C_{\mathcal{E}}$ are defined in (4.8), (4.10), (4.19) and (4.21).

Thanks to estimates (3.17) and (4.2), we have immediately the following polynomial decay results.

Theorem 4.2. Assume that all conditions of Theorem 4.1 are satisfied. Let $u(x, t)$ be the global solution of system (2.1). Then we have for all $t \geq 0$,

$$
\begin{aligned}
& 0<\|\nabla u(t)\|^{2} \leq \frac{\left[(2 \gamma+2) C_{E}\right]^{\frac{1}{\gamma+1}}}{(1+t)^{\frac{2}{\gamma}}}, \\
& \left\|u^{\prime}(t)\right\|^{2} \leq \frac{2 C_{E}}{(1+t)^{\frac{2 \gamma+2}{\gamma}}}, \\
& \|\Delta u(t)\|^{2} \leq \frac{(2 \gamma+2) \lambda \kappa C_{E}}{(1+t)^{\frac{2 \gamma+2}{\gamma}}}, \\
& \left\|\nabla u^{\prime}(t)\right\|^{2} \leq \frac{\lambda \kappa\left[(2 \gamma+2) C_{E}\right]^{\frac{2 \gamma+1}{\gamma+1}}}{(1+t)^{\frac{4 \gamma+2}{\gamma}}},
\end{aligned}
$$

where $C_{E}$ is defined in Remark 4.1, and $\lambda$ and $\kappa$ are defined in Remark 3.1.

Proof of Theorem 4.1. We first set

$$
\tilde{\rho}(t) \doteq 2\left(u^{\prime}(t), m \cdot \nabla u(t)\right)+(n-1)\left(u^{\prime}(t), u(t)\right) .
$$


Similar to the proof of Lemma 3.2, we multiply the first equation of system (2.1) by $[2 m \cdot \nabla u(t)+(n-1) u(t)](E(t))^{p}(p>0)$,

$$
\begin{aligned}
(E(t))^{p} & \left(\left\|u^{\prime}(t)\right\|^{2}+\|\nabla u(t)\|^{2 \gamma+2}\right)+\frac{d}{d t}\left[(E(t))^{p} \tilde{\rho}(t)\right] \\
= & (E(t))^{p}\|\nabla u(t)\|_{\Omega}^{2 \gamma} \int_{\Gamma_{0}}(m \cdot \nu)|\nabla u(t)|^{2} d \Gamma+(E(t))^{p} \int_{\Gamma_{1}}(m \cdot \nu)\left|u^{\prime}(t)\right|^{2} d \Gamma \\
& +(E(t))^{p}\|\nabla u(t)\|_{\Omega}^{2 \gamma} \int_{\Gamma_{1}}\left[-(m \cdot \nu)|\nabla u(t)|^{2}+2 \partial_{\nu} u(t)(m \cdot \nabla u(t))\right. \\
& \left.+(n-1) \partial_{\nu} u(t) u(t)\right] d \Gamma+\tilde{\rho}(t) \frac{d}{d t}(E(t))^{p} .
\end{aligned}
$$

Thus, by using assumptions (B1), (B2), (2.3) and the Cauchy-Schwarz inequality,

$$
\begin{aligned}
2(E(t))^{p+1}+\frac{d}{d t}\left[(E(t))^{p} \tilde{\rho}(t)\right] \\
\leq(E(t))^{p}\left[R\left|u^{\prime}(t)\right|_{\Gamma_{1}}^{2}+\frac{R^{2}}{m_{0}}\|\nabla u(t)\|_{\Omega}^{2 \gamma}\left|\partial_{\nu} u(t)\right|_{\Gamma_{1}}^{2}\right. \\
\left.\quad+(n-1)\|\nabla u(t)\|_{\Omega}^{2 \gamma}\left\langle\partial_{\nu} u(t), u(t)\right\rangle_{\Gamma_{1}}\right]-(E(t))^{p-1} \tilde{\rho}(t)\left\langle g\left(u^{\prime}(t)\right), u^{\prime}(t)\right\rangle_{\Gamma_{1}} .
\end{aligned}
$$

From $(\mathrm{G})$, the third equation of (2.1), (2.14) and (4.5), we have

$$
\begin{aligned}
& \frac{3}{2}(E(t))^{p+1}+\frac{d}{d t}\left[(E(t))^{p} \tilde{\rho}(t)\right] \\
& \quad \leq R(E(t))^{p}\left|u^{\prime}(t)\right|_{\Gamma_{1}}^{2}+c_{4}(E(t))^{p-1}|\tilde{\rho}(t)|\left|u^{\prime}(t)\right|_{\Gamma_{1}}^{2}+\frac{\eta(E(t))^{p}\left|u^{\prime}(t)\right|_{\Gamma_{1}}^{2}}{\|\nabla u(t)\|_{\Omega}^{2 \gamma}},
\end{aligned}
$$

where

$$
\eta \doteq \frac{c_{4}^{2} R^{2}}{m_{0}}+c_{4}^{2} C_{\Omega}(n-1)^{2}(\gamma+1) .
$$

Furthermore, it is clear that

$$
|\tilde{\rho}(t)| \leq \sqrt{2}(2 R+n-1)(2 \gamma+2)^{\frac{1}{2 \gamma+2}}(E(t))^{\frac{\gamma+2}{2 \gamma+2}} \doteq \tilde{\rho}_{0}(E(t))^{\frac{\gamma+2}{2 \gamma+2}} .
$$

Therefore, by (2.3), (4.6) and (4.8),

$$
\begin{aligned}
& \frac{3}{2}(E(t))^{p+1}+\frac{d}{d t}\left[(E(t))^{p} \tilde{\rho}(t)\right] \\
& \quad \leq\left[R(E(0))^{1-\frac{\gamma+2}{2 \gamma+2}}+c_{4} \tilde{\rho}_{0}\right](E(t))^{p-1+\frac{\gamma+2}{2 \gamma+2}}\left|u^{\prime}(t)\right|_{\Gamma_{1}}^{2}+\frac{\eta(E(t))^{p}\left|u^{\prime}(t)\right|_{\Gamma_{1}}^{2}}{\|\nabla u(t)\|_{\Omega}^{2 \gamma}} .
\end{aligned}
$$

Set

$$
\tilde{\mathcal{E}}(t) \doteq \tilde{\lambda}(E(t))^{p+\frac{\gamma+2}{2 \gamma+2}}+(E(t))^{p} \tilde{\rho}(t)
$$

where

$$
\tilde{\lambda}>2 \max \left\{\tilde{\rho}_{0}, c_{3}^{-1}\left(p+\frac{\gamma+2}{2 \gamma+2}\right)^{-1}\left[R(E(0))^{1-\frac{\gamma+2}{2 \gamma+2}}+c_{4} \tilde{\rho}_{0}\right]\right\} .
$$

Then, from (4.8),

$$
\left(\tilde{\lambda}-\tilde{\rho}_{0}\right)(E(t))^{p+\frac{\gamma+2}{2 \gamma+2}} \leq \tilde{\mathcal{E}}(t) \leq\left(\tilde{\lambda}+\tilde{\rho}_{0}\right)(E(t))^{p+\frac{\gamma+2}{2 \gamma+2}} .
$$


Notice that by $(\mathrm{G})$,

$$
\frac{d}{d t}(E(t))^{p+\frac{\gamma+2}{2 \gamma+2}} \leq-c_{3}\left(p+\frac{\gamma+2}{2 \gamma+2}\right)(E(t))^{p-1+\frac{\gamma+2}{2 \gamma+2}}\left|u^{\prime}\right|_{\Gamma_{1}}^{2} .
$$

Therefore, we have by (4.9) and (4.12) that

$$
\frac{3}{2}(E(t))^{p+1}+\frac{d}{d t} \tilde{\mathcal{E}}(t) \leq \frac{\eta(E(t))^{p}\left|u^{\prime}(t)\right|_{\Gamma_{1}}^{2}}{\|\nabla u(t)\|_{\Omega}^{2 \gamma}} .
$$

Moreover, it follows from (3.17) that

$$
\frac{\left\|\nabla u^{\prime}(t)\right\|^{2}}{\|\nabla u(t)\|^{4 \gamma+2}} \leq H_{2}(t) \leq \lambda \mathcal{E}(0), \quad \forall t \geq 0
$$

Consequently,

$$
\frac{\left|u^{\prime}(t)\right|_{\Gamma_{1}}^{2}}{\|\nabla u(t)\|^{2 \gamma}} \leq \lambda C_{\Omega}(2 \gamma+2) \mathcal{E}(0) E(t), \quad \forall t \geq 0 .
$$

Replacing (4.15) into (4.13) yields that

$$
\frac{3}{2}(E(t))^{p+1}+\frac{d}{d t} \tilde{\mathcal{E}}(t) \leq \eta \lambda C_{\Omega}(2 \gamma+2) \mathcal{E}(0)(E(t))^{p+1} .
$$

Thus, it follows from Remark 4.1 and (4.16) that

$$
(E(t))^{p+1}+\frac{d}{d t} \tilde{\mathcal{E}}(t) \leq 0 .
$$

From (4.11) and (4.17), we obtain that

$$
\left[\left(\tilde{\lambda}+\tilde{\rho}_{0}\right)^{-1} \tilde{\mathcal{E}}(t)\right]^{\theta}+\frac{d}{d t} \tilde{\mathcal{E}}(t) \leq 0
$$

where

$$
\theta \doteq \frac{p+1}{p+\frac{\gamma+2}{2 \gamma+2}}>1
$$

By (4.18), there exists a positive constant $C_{\mathcal{E}}$ such that

$$
(\tilde{\mathcal{E}}(t))^{\theta-1} \leq \frac{C_{\mathcal{E}}}{1+t},
$$

where

$$
C_{\mathcal{E}} \doteq \frac{1}{\min \left\{\left(\tilde{\lambda}+\tilde{\rho}_{0}\right)^{-\theta}(\theta-1),(\tilde{\mathcal{E}}(0))^{1-\theta}\right\}} .
$$

Thus, the desired result is reached from (4.11) and (4.20).

REMARK 4.2. In [12], Lasiecka and Ong obtained the exponential stability of the solution of the nondegenerate system when $g(\cdot)$ satisfies $(\mathrm{G})$. For the degenerate case, since the auxiliary function $\tilde{\rho}(t)$ is not of the same order as $E(t)$ by (4.8), we can only reach the polynomial decay of the solution. On the other hand, we cannot estimate the tangential trace in (4.4) by Lemma 1 because of the multiplier $(E(t))^{p}$. Therefore, we need the geometry condition (B2) in Theorem 4.1 . 
5. Appendix. In this section we prove Lemma 3.2. Denote $w(x, t)=u^{\prime}(x, t)$. Then $w(x, t)$ satisfies the following system:

$$
\left\{\begin{array}{lll}
w^{\prime \prime}-\|\nabla u\|^{2 \gamma} \Delta w-\frac{2 \gamma\left(\nabla u, \nabla u^{\prime}\right) w^{\prime}}{\|\nabla u\|^{2}}=0 & \text { in } & \Omega \times\left[0, T_{1}\right), \\
w=0 & \text { on } & \Gamma_{0} \times\left[0, T_{1}\right), \\
\partial_{\nu} w=-\frac{g^{\prime}(w) w^{\prime}}{\|\nabla u\|^{2 \gamma}}+\frac{2 \gamma\left(\nabla u, \nabla u^{\prime}\right) g(w)}{\|\nabla u\|^{2 \gamma+2}} & \text { on } & \Gamma_{1} \times\left[0, T_{1}\right) .
\end{array}\right.
$$

To prove Lemma 3.2, we need the following estimate of the tangential trace $\left|\partial_{\tau} w\right|_{\Gamma_{1}}^{2}$.

Lemma 1 (11, 12]). Let $w(x, t)$ satisfy system (5.1) and $0<t<T_{1}$. Then for any $0<\delta<\frac{t}{2}$ and $\epsilon, \epsilon_{0}>0$, there exist positive constants $C_{\delta, \epsilon}, C_{\delta, \epsilon_{0}}$ such that the following trace estimate holds:

$$
\begin{aligned}
& \int_{\delta}^{t-\delta}\left|\partial_{\tau} w(s)\right|_{\Gamma_{1}}^{2} d s \leq C_{\delta, \epsilon} \int_{0}^{t}\left(\left|\partial_{\nu} w(s)\right|_{\Gamma_{1}}^{2}+\left|w^{\prime}(s)\right|_{\Gamma_{1}}^{2}+4 \gamma^{2} \Phi_{1}^{2}(s)\left\|w^{\prime}(s)\right\|_{H^{-\frac{1}{2}+\epsilon}(\Omega)}^{2}\right) d s \\
& \quad+C_{\delta, \epsilon_{0}}\|w(\cdot)\|_{H^{\frac{1}{2}+\epsilon_{0}}((0, t) \times \Omega)}^{2}
\end{aligned}
$$

By using the multiplier method and Lemma 1, we obtain the following inequality.

Lemma 2. Assume (B1), (G) hold and $\gamma \geq 1$. Let $w(x, t)$ satisfy system (5.1) and $0<t<T_{1}$. Then for any $0<\delta<\frac{t}{2}$ and $\epsilon, \epsilon_{0}>0$, there exist positive constants $C_{\delta, \epsilon}, C_{\delta, \epsilon_{0}}$ such that

$$
\begin{aligned}
& \int_{0}^{t} H_{1} d s \leq 2 \delta H_{1}(0)+2 \rho_{0} H_{2}(0)+\int_{0}^{t}\left[\frac{R\left|w^{\prime}\right|_{\Gamma_{1}}^{2}}{\|\nabla u\|^{2 \gamma}}+2 R C_{\delta, \epsilon}\left|w^{\prime}\right|_{\Gamma_{1}}^{2}+\frac{|w|_{\Gamma_{1}}^{2}}{4 C_{\Omega}}\right. \\
& \quad+\left(3 R+2 R C_{\delta, \epsilon}+(n-1)^{2} C_{\Omega}\right)\left|\partial_{\nu} w\right|_{\Gamma_{1}}^{2}+\frac{8 \gamma^{2}}{c_{1}}\left(\delta \Phi_{\gamma+1}^{2}+\rho_{0} \Phi_{3 \gamma+2}^{2}\right)|g(w)|_{\Gamma_{1}}^{2} \\
& \left.\quad+4 \gamma \delta \Phi_{1} H_{1}+2(4 \gamma+2) \rho_{0} \Phi_{1} H_{2}+8 \gamma^{2} R C_{\delta, \epsilon} \Phi_{1}^{2}\left\|w^{\prime}\right\|_{H^{-\frac{1}{2}+\epsilon}(\Omega)}^{2}\right] d s \\
& \quad+2 R C_{\delta, \epsilon_{0}}\|w\|_{H^{\frac{1}{2}+\epsilon_{0}}((0, t) \times \Omega)}^{2}
\end{aligned}
$$

Proof. For $0<S \leq T<T_{1}$, we multiply the first equation of (5.1) by $\frac{m \cdot \nabla w}{\|\nabla u\|^{2 \gamma}}$ and integrate it on $\Omega \times[S, T]$ to get

$$
\begin{aligned}
& \left.\frac{\left(w^{\prime}, m \cdot \nabla w\right)}{\|\nabla u\|^{2 \gamma}}\right|_{s=S} ^{T}+\int_{S}^{T}\left[\frac{n}{2} \frac{\left\|w^{\prime}\right\|^{2}}{\|\nabla u\|^{2 \gamma}}+\left(1-\frac{n}{2}\right)\|\nabla w\|^{2}\right] d s \\
& =\frac{1}{2} \int_{S}^{T} \int_{\Gamma_{0}}(m \cdot \nu)|\nabla w|^{2} d \Gamma d s+\frac{1}{2} \int_{S}^{T} \int_{\Gamma_{1}} \frac{(m \cdot \nu)\left|w^{\prime}\right|^{2}}{\|\nabla u\|^{2 \gamma}} d \Gamma d s \\
& \quad-\frac{1}{2} \int_{S}^{T} \int_{\Gamma_{1}}(m \cdot \nu)|\nabla w|^{2} d \Gamma d s+\int_{S}^{T} \int_{\Gamma_{1}} \partial_{\nu} w(m \cdot \nabla w) d \Gamma d s .
\end{aligned}
$$


Furthermore, multiplying the first equation of (5.1) by $\frac{w}{\|\nabla u\|^{2 \gamma}}$, we get that

$$
\left.\frac{\left(w^{\prime}, w\right)}{\|\nabla u\|^{2 \gamma}}\right|_{s=S} ^{T}+\int_{S}^{T}\left(\|\nabla w\|^{2}-\frac{\left\|w^{\prime}\right\|^{2}}{\|\nabla u\|^{2 \gamma}}\right) d s=\int_{S}^{T}\left\langle\partial_{\nu} w, w\right\rangle_{\Gamma_{1}} d s .
$$

From (5.4) and (5.5), we have

$$
\begin{aligned}
\rho(T) & -\rho(S)+\int_{S}^{T}\left(\frac{\left\|w^{\prime}\right\|^{2}}{\|\nabla u\|^{2 \gamma}}+\|\nabla w\|^{2}\right) d s \\
= & \int_{S}^{T} \int_{\Gamma_{0}}(m \cdot \nu)|\nabla w|^{2} d \Gamma d s+\int_{S}^{T} \int_{\Gamma_{1}} \frac{(m \cdot \nu)\left|w^{\prime}\right|^{2}}{\|\nabla u\|^{2 \gamma}} d \Gamma d s-\int_{S}^{T} \int_{\Gamma_{1}}(m \cdot \nu)|\nabla w|^{2} d \Gamma d s \\
& +\int_{S}^{T} \int_{\Gamma_{1}} \partial_{\nu} w[2(m \cdot \nabla w)+(n-1) w] d \Gamma d s .
\end{aligned}
$$

Thus, by using assumption (B1) in (5.6),

$$
\begin{gathered}
\int_{S}^{T} H_{1} d s \leq|\rho(T)|+|\rho(S)|+\int_{S}^{T}\left[\frac{R\left|w^{\prime}\right|_{\Gamma_{1}}^{2}}{\|\nabla u\|^{2 \gamma}}+\frac{|w|_{\Gamma_{1}}^{2}}{4 C_{\Omega}}+2 R\left|\partial_{\tau} w\right|_{\Gamma_{1}}^{2}\right. \\
\left.+\left(3 R+(n-1)^{2} C_{\Omega}\right)\left|\partial_{\nu} w\right|_{\Gamma_{1}}^{2}\right] d s
\end{gathered}
$$

where $C_{\Omega}$ is the constant defined in (2.14). From (5.2) and (5.7),

$$
\begin{aligned}
& \int_{\delta}^{t-\delta} H_{1} d s \leq|\rho(t-\delta)|+|\rho(\delta)|+\int_{\delta}^{t-\delta}\left[\frac{R\left|w^{\prime}\right|_{\Gamma_{1}}^{2}}{\|\nabla u\|^{2 \gamma}}+\frac{|w|_{\Gamma_{1}}^{2}}{4 C_{\Omega}}+2 R C_{\delta, \epsilon}\left|w^{\prime}\right|_{\Gamma_{1}}^{2}+\left(3 R+2 R C_{\delta, \epsilon}\right.\right. \\
& \left.\left.+(n-1)^{2} C_{\Omega}\right)\left|\partial_{\nu} w\right|_{\Gamma_{1}}^{2}+8 \gamma^{2} R C_{\delta, \epsilon} \Phi_{1}^{2}\left\|w^{\prime}\right\|_{H^{-\frac{1}{2}+\epsilon}(\Omega)}^{2}\right] d s+2 R C_{\delta, \epsilon_{0}}\|w\|_{H^{\frac{1}{2}+\epsilon_{0}}((0, t) \times \Omega)}^{2}
\end{aligned}
$$

Moreover, we have from (3.6) that

$$
\int_{0}^{\delta} H_{1} d s+\int_{t-\delta}^{t} H_{1} d s \leq 2 \delta H_{1}(0)+2 \delta \int_{0}^{t}\left(\frac{4 \gamma^{2}}{c_{1}} \Phi_{\gamma+1}^{2}|g(w)|_{\Gamma_{1}}^{2}+2 \gamma \Phi_{1} H_{1}\right) d s .
$$

By (3.4) and (3.7),

$$
|\rho(t-\delta)|+|\rho(\delta)| \leq 2 \rho_{0} H_{2}(0)+2 \rho_{0} \int_{0}^{t}\left[\frac{4 \gamma^{2}}{c_{1}} \Phi_{3 \gamma+2}^{2}|g(w)|_{\Gamma_{1}}^{2}+(4 \gamma+2) \Phi_{1} H_{2}\right] d s .
$$

Therefore, we get the desired result from (5.8)-(5.10).

Now we give the estimate for the last term in (5.3).

Lemma 3. Assume $\gamma \geq 1$. Let $w(x, t)$ satisfy system (5.1) and $0<t<T_{1}$. Then there exists a constant $C_{t}$ such that

$$
\|w\|_{H^{\frac{1}{2}+\epsilon_{0}((0, t) \times \Omega)}}^{2} \leq C_{t}\left[\int_{0}^{t}\left|\partial_{\nu} w\right|_{\Gamma_{1}}^{2} d s+\|\nabla w(0)\|^{2}+\left\|w^{\prime}(0)\right\|^{2}\right] .
$$

Proof. Suppose there exists a sequence $\left\{w_{n}\right\}$ satisfying (5.1) such that

$$
\left\|w_{n}\right\|_{H^{\frac{1}{2}+\epsilon_{0}((0, t) \times \Omega)}}^{2}=1, \quad \lim _{n \rightarrow \infty}\left[\int_{0}^{t}\left|\partial_{\nu} w_{n}\right|_{\Gamma_{1}}^{2} d s+\left\|\nabla w_{n}(0)\right\|^{2}+\left\|w_{n}^{\prime}(0)\right\|^{2}\right]=0 .
$$


Then, there exist $\tilde{w}_{0} \in H^{1}(\Omega)$ and $\tilde{w}_{1} \in L^{2}(\Omega)$,

$$
\begin{array}{ll}
w_{n}(0) \rightarrow \tilde{w}_{0} & \text { weakly in } H^{1}(\Omega), \\
w_{n}^{\prime}(0) \rightarrow \tilde{w}_{1} & \text { weakly in } L^{2}(\Omega) .
\end{array}
$$

Denote by $\tilde{w}$ the solution of (5.1) with initial data $\tilde{w}_{0}, \tilde{w}_{1}$. Then,

$$
\begin{array}{ll}
w_{n} \rightarrow \tilde{w} & \text { weak star in } L^{\infty}\left((0, t), H^{1}(\Omega)\right), \\
w_{n}^{\prime} \rightarrow \tilde{w}^{\prime} & \text { weak star in } L^{\infty}\left((0, t), L^{2}(\Omega)\right) .
\end{array}
$$

Therefore, by [18],

$$
w_{n} \rightarrow \tilde{w} \quad \text { strongly in } H^{\frac{1}{2}+\epsilon_{0}}((0, t) \times \Omega) .
$$

Thus, $\tilde{w}$ satisfies (5.1) and

$$
\begin{gathered}
\|\tilde{w}\|_{H^{\frac{1}{2}+\epsilon_{0}}((0, t) \times \Omega)}^{2}=1, \\
\int_{0}^{t}\left|\partial_{\nu} \tilde{w}\right|_{\Gamma_{1}}^{2} d s=0 .
\end{gathered}
$$

However, by (5.1) and (5.19), we have $\tilde{w}=0$ in $(0, t) \times \Omega$, which contradicts (5.18).

By Lemma 3 and (2.3), we have that

$$
\|w\|_{H^{\frac{1}{2}+\epsilon_{0}((0, t) \times \Omega)}}^{2} \leq C_{t} \int_{0}^{t}\left|\partial_{\nu} w\right|_{\Gamma_{1}}^{2} d s+C_{t}\left(1+e_{0}^{2 \gamma}\right) H_{1}(0) .
$$

Therefore, Lemma 3.2 is proved by combining Lemma 2 with (5.20).

Acknowledgements. The author would like to thank the referees for helpful suggestions.

\section{REFERENCES}

[1] A. Arosio and S. Garavaldi, On the mildly degenerate Kirchhoff string, Mathematical Methods in Applied Science, 14 (1991), 177-195. MR1099324 (92c:35072)

[2] V. Barbu, I. Lasiecka, and M. A. Rammaha, On nonlinear wave equations with degenerate damping and source terms, Transactions of the American Mathematical Society, 357 (2005), 2571-2611. MR2139519 (2006a:35203)

[3] E. H. de Brito, Decay estimates for the generalized damped extensible string and beam equation, Nonlinear Analysis, 8 (1984), 1489-1496. MR769410 (86k:34056)

[4] G.F. Carrier, On the non-linear vibration problem of the elastic string, Quarterly of Applied Mathematics, 3 (1945), 157-165. MR0012351(7:13h)

[5] M. M. Cavalcanti, U V. N. Domingos Cavalcanti, J. S. Prates Filho, and J. A. Soriano, Existence and exponential decay for a Kirchhoff-Carrier model with viscosity, Journal of Mathematical Analysis and Applications, 226 (1998), 40-60. MR1646453 (99g:35084)

[6] P. D'Ancona and S. Spagnolo, Nonlinear perturbations of the Kirchhoff equation, Communications on Pure and Applied Mathematics, 47 (1994), 1005-1029. MR.1283880 (95d:35105)

[7] M. Ghisi, Global solutions for dissipative Kirchhoff strings with non-Lipschitz nonlinear term, Journal of Differential Equations, 230 (2006), 128-139. MR2270549(2008a:35196)

[8] R. Izaguirrea, R. Fuentesb and M. M. Miranda, Existence of local solutions of the Kirchhoff-Carrier equation in Banach spaces, Nonlinear Analysis, 68 (2008), 3565-3580. MR2401368 (2009d:35233)

[9] G. Kirchhoff, Vorlesungen über Mechanik, Teubner, Leipzig, 1883.

[10] V. Komornik, Exact Controllability and Stabilization: The Multiplier Method, Masson, Paris, John Wiley and Sons, Chichester, 1994. MR:1359765 (96m:93003) 
[11] I. Lasiecka and R. Triggiani, Uniform stabilization of the wave equation with Dirichlet or Neumann feedback control without geometric conditions, Applied Mathematics and Optimization, 25 (1992), 189-224. MR.1142681 (93b:93099)

[12] I. Lasiecka and J. Ong, Global solvability and uniform decays of solutions to quasilinear equation with nonlinear boundary dissipation, Communications in Partial Differential Equations, 24 (1999), 2069-2107. MR.1720766 (2001c:35154)

[13] J. L. Lions, On some questions in boundary value problem of mathematical physics, in: G.M. de La Penha, L.A. Medeiros (Eds.), Contemporary development in continuum mechanics and partial differential equations, North-Holland, Amsterdam, 1978, 285-346. MR519648 (82b:35020)

[14] K. Nishihara, Global existence and asymptotic behaviour of the solution of some quasilinear hyperbolic equation with linear damping, Funkcialaj Ekvacioj, 32 (1989), 343-355. MR1040163 (91h:35213)

[15] K. Nishihara and Y. Yamada, On global solutions of some degenerate quasilinear hyperbolic equations with dissipative terms, Funkcialaj Ekvacioj, 33 (1990), 151-159. MR1065473 (91f:35181)

[16] K. Ono, Global existence, decay, and blowup of solutions for some mildly degenerate nonlinear Kirchhoff strings, Journal of Differential Equations 137 (1997), 273-301. MR1456598 (98f:35149)

[17] M. L. Santos, J. Ferreira, D. C. Pereira and C. A. Raposo, Global existence and stability for wave equation of Kirchhoff type with memory condition at the boundary, Nonlinear Anaysis, 54 (2003), 959-976. MR.1992515 (2004e:35152)

[18] J. Simon, Compact sets in the space $L^{p}(0, T ; B)$, Annali di Matematica pura ed applicata, 146 (1987), 65-96. MR916688 (89c:46055)

[19] R. Temam, Infinite Dimensional Dynamical Systems in Mechanics and Physics, Springer-Verlag, Applied Mathematical Sciences, Vol. 68, New York, 1988. MR.953967 (89m:58056)

[20] Q. Zhang, Global solution for a quasi-linear plate system with boundary memory damping, IMA Journal of Applied Mathematics, 2009. MR2507295 (2010j:35553) 